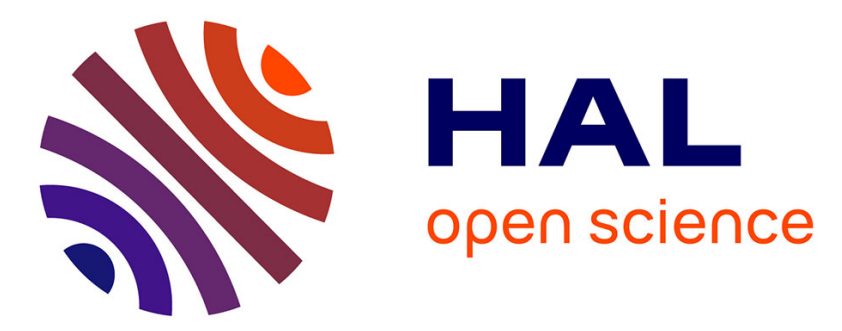

\title{
Quality-delay tradeoff for video streaming over mobile ad hoc networks
}

Liang Zhou, Yan Zhang, Joel Rodrigues, Benoit Geller, Jingwu Cui, Baoyu Zheng

\section{- To cite this version:}

Liang Zhou, Yan Zhang, Joel Rodrigues, Benoit Geller, Jingwu Cui, et al.. Quality-delay tradeoff for video streaming over mobile ad hoc networks. ICC, 2012, Ottawa, Canada. 10.1109/ICC.2012.6363798 . hal-01225548

\section{HAL Id: hal-01225548 \\ https://hal.science/hal-01225548}

Submitted on 4 Dec 2015

HAL is a multi-disciplinary open access archive for the deposit and dissemination of scientific research documents, whether they are published or not. The documents may come from teaching and research institutions in France or abroad, or from public or private research centers.
L'archive ouverte pluridisciplinaire $\mathbf{H A L}$, est destinée au dépôt et à la diffusion de documents scientifiques de niveau recherche, publiés ou non, émanant des établissements d'enseignement et de recherche français ou étrangers, des laboratoires publics ou privés. 


\title{
Quality-Delay Tradeoff for Video Streaming over Mobile Ad Hoc Networks
}

\author{
Liang Zhou ${ }^{\dagger}$, Yan Zhang ${ }^{\ddagger}$, Joel Rodrigues ${ }^{\curvearrowright}$, Benoit Geller ${ }^{\curvearrowright}$, Jingwu Cui ${ }^{\dagger}$, Baoyu Zheng ${ }^{\dagger}$ \\ ${ }^{\dagger}$ Key Lab of Broadband Wireless Communication and Sensor Network Technology (Nanjing University of Posts \\ and Telecommunications), Ministry of Education, China \\ ${ }^{\ddagger}$ Simula Research Laboratory, Norway \\ ${ }^{\ominus}$ Instituto de Telecomunicaçőes, University of Beira Interior, Portugal \\ $\diamond_{\text {Lab. of UEI, ENSTA-ParisTech, France }}$
}

\begin{abstract}
In this work, we study the quality-delay tradeoff for video streaming over mobile ad hoc network by utilizing a class of scheduling schemes. We show that node spatial mobility indeed impacts on the performance of wireless video transmission under the assumption that all the nodes can identically and uniformly visit the entire network. To describe a practical mobile scenario, we consider a random walk mobility model in which each node can randomly and independently choose its mobility direction at each time-slot. The contributions of this work are twofold: 1) It investigates the optimal node velocity for the mobile video network which helps to identify the impact of mobility on the video performance; 2) It derives the achievable quality-delay tradeoff range for any node mobility velocity, and thus it is helpful to design appropriate quality and delay requirements. These results provide insights on network design and fundamental guidelines on establishing an efficient mobile wireless video transmission system.
\end{abstract}

Index Terms-Wireless ad hoc network; video transmission; quality; delay; tradeoff.

\section{INTRODUCTION}

$\mathbf{I}$ $\mathrm{N}$ recent years, there has been a tremendous increase in demand for video communications over mobile ad hoc networks, and the corresponding techniques can fall into three categories [1]-[5]: 1) designing efficient media-aware routing, scheduling and resource allocation algorithms in the framework of cross-layer design; 2) developing novel source/channel coding algorithms aiming at adapting video frames to optimal bit-rate streams, such as multiple description coding, scalable video coding; 3) providing pervasive multimedia service environment by combining an adaptive service provisioning with a context-aware multimedia middleware.

In contrast to the abundance of the above mentioned techniques, few literature focuses on how the mobility, e.g., the direction and velocity of the node mobility, impacts the performance of video streaming over wireless networks. Fig. 1 presents the results of a simulation study that compares the video quality (in terms of PSNR) with different node velocities. Specifically, the simulation settings and video scheduling schemes are the same with that of $[3]^{1}$. All the nodes follows a random walk mobility model, i.e., they can randomly and independently choose the mobility direction at each time-slot

\footnotetext{
${ }^{1}$ Since this paper does not consider a multi-channel scenario, for fair comparison, we set the channel number as 1 in [3].
}

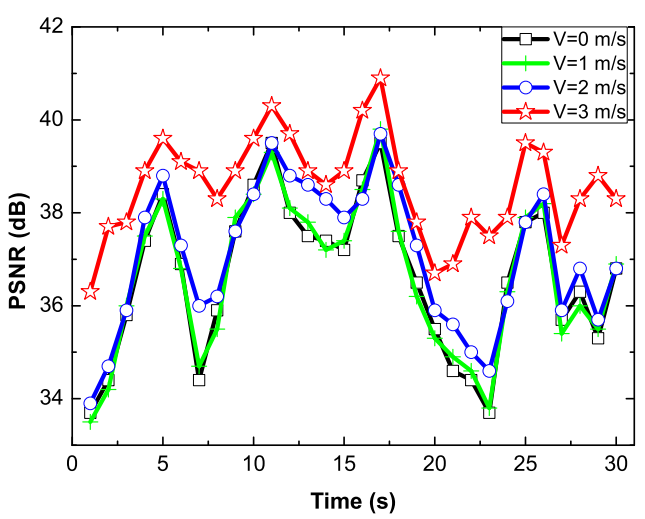

Fig. 1. Video quality comparison for wireless ad hoc networks with different node velocities.

(the precise definition of this model is presented in Section II). From the given results, we can observe that: compared to PSNR value of the node immobility case $(V=0 \mathrm{~m} / \mathrm{s})$, $V=1 \mathrm{~m} / \mathrm{s}$ gets almost the same performance, $V=2 \mathrm{~m} / \mathrm{s}$ is better in some extent, and $V=3 \mathrm{~m} / \mathrm{s}$ is consistently and dramatically better. This is an interesting phenomenon which motivates us to investigate the properties of wireless video transmission in the context of mobile environment, preferably in a quantitative manner.

The objective of this work is to make a pace towards clarifying how the node mobility impacts video streaming over wireless ad hoc networks. We bridge the theoretical analysis of fundamental disciplines of mobile video communications with the development of distributed wireless video scheduling. Under what conditions is it possible to achieve the optimal video quality given the transmission delay constraints for the mobile ad hoc networks? How efficiently can video streams be transmitted in the network? And to what extent does one can achieve the quality-delay tradeoff range? These questions are the main focus of this work.

The rest of this paper is organized as follows. In Section II, we present corresponding system models for video streaming over mobile ad hoc networks. In section III, we summarize our main results on the achievable quality-delay tradeoff range for 


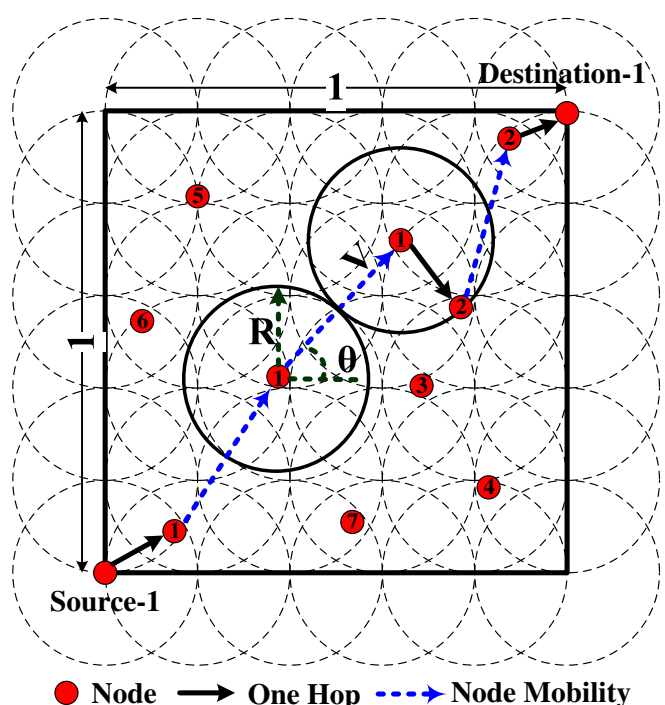

Fig. 2. Node random walk mobility model.

each velocity, and the rigorous proofs are given in Section IV. Finally, we conclude with a discussion of the contributions in Section V.

\section{SYSTEM MODEL}

\section{A. Mobility Model}

There are $\mathcal{N}=\{1, \ldots, n, \ldots, N\}(N \in \mathbb{N})$ nodes which are positioned in a unit torus square, i.e., the left and right edges are assumed to touch each other and the top and bottom edges are also connected to each other. Without loss of generality, each node is both a source and a destination, and all the nodes can identically and uniformly visit the entire network area. Each node's transmission range is $R\left(R \in \mathbb{R}_{+}\right)$, which is a constant parameter in this work. The video transmission delay is divided into some time-slots with unit length. In order to depict a practical mobile network scenario, we consider a general Random Walk Mobility Model (RWMM). Specifically, at the beginning of each time-slot, each node uniformly select a random mobility direction $\theta \in[0,2 \pi)$. Then, the node moves along this direction with a constant velocity $V(V \in$ $\mathbb{R}_{+}$) during the rest of time-slot. The mobility directions of the nodes can be selected again after each time-slot, totally independently from time-slot and node. Fig. 2 illustrates the behavior of RWMM.

\section{B. Network Model}

There are $\mathcal{Z}=\{1, \ldots, z, \ldots, Z\}$ video flows in the networks, for each flow $z$, it can be categorized into one of $K$ classes (i.e., $\left.C_{1}, \ldots, C_{K}\right)$. In addition, a class $C_{k}$ can be modeled as a triplet $\left(D_{k}, W_{k}, \lambda_{k}\right): D_{k}$ represents the delay deadline of $C_{k} ; W_{k}$ is the average source rate of each flow in $C_{k} ; \lambda_{k}$ denotes the quality impact factor of $C_{k}$. We refer the interested readers to [4] for more details on how these parameters can be extracted.

Let $\mathcal{E}$ be the available link set in the wireless networks, and each link $l \in \mathcal{E}$ is a memoryless packet erasure channel.
IEEE 802.11a standard is deployed to access the channel. We denote the packet loss rate over link $l$ for a flow in class $C_{k}$ as $p_{l, k}$, which can be approximated using the sigmoid function [4]. Moreover, we denote $T_{l, k}$ to represent the maximum transmission rate for class $C_{k}$ streaming over link $l$. According to [3], the effective transmission rate for flow $z$ transmitting over link $l$ can be computed by $T_{l, k}\left(1-p_{l, k}\right) t_{l, z}$, where $t_{l, z}$ shows the time sharing fraction for video flow $z$ streaming over link $l$. In particular, $t_{l, z}=0$ shows that flow $z$ does not stream over link $l$.

\section{Video Scheduling}

We define the scheduling of a video flow $z$ as $x_{z}=\left\{t_{l, z}\right\}$, and let $\mathcal{X}=\left[x_{1}, x_{2}, \ldots x_{Z}\right]$ be the joint scheduling for all $\mathcal{Z}$ video flows. $D_{z}(\mathcal{X})$ represents the delay for transmitting the flow $z$ from source to destination based on $\mathcal{X}$. According to previous models, $D_{z}(\mathcal{X})$ can be given by [4]:

$$
D_{z}(\mathcal{X})=\sum_{l, t_{l, z}>0} \frac{L_{k}}{t_{l, z}\left(1-p_{l, k}\right) T_{l, k}}, z \in C_{k},
$$

where $L_{k}$ is the average packet size of $C_{k}$. Therefore, the received video quality from node $n$, denoted by $Q_{n}$, can be expressed as:

$$
Q_{n}(\mathcal{X})=\sum_{k=1}^{K} \sum_{z=1}^{N_{n k}} \lambda_{k} \cdot W_{k} \cdot I\left(D_{z}(\mathcal{X}) \leq D_{k}\right),
$$

where $N_{n k}$ is the number of flows in class $C_{k}$ from $n$ and $I(\cdot)$ is the indicator function [4]. Therefore, the wireless video scheduling can be formulated as

$$
\begin{gathered}
\arg \max _{\mathcal{X}}\left\{\sum_{n=1}^{N} Q_{n}(\mathcal{X})\right\}, \\
\text { s.t. } \quad \sum_{z=1}^{Z} t_{l, z} \leq 1, \forall l \in \mathcal{E}, \\
D_{z}(\mathcal{X}) \leq D_{k}, \forall z \in C_{k}, z=1, \ldots, Z, k=1, \ldots, K .
\end{gathered}
$$

Specifically, (3.1) is the resource constraint for each link, and (3.2) is the delay constraint for each flow. In this paper, we employ the Distributed Minimum-Distortion Scheduling (DMDS) introduced in [3] as our operation rule ${ }^{2}$. It can be viewed as a member of the distortion-aware scheduling family. In short, DMDS studies the tradeoff between video quality and transmission delay the two competing objectives in a convex optimization formulation, and discusses the distributed solution by jointly utilizing multi-path routing and cross-layer design.

\section{MAIN RESUlTS}

Recall that we aim at studying the impact of velocity $V$ on the average video quality $Q$ and on the average transmission delay $D$ in the context of wireless ad hoc networks with RWMM. In particular, we define the $Q$ and $D$ as: $Q \triangleq$ $\frac{\sum_{n=1}^{N} Q_{n}}{N}$ and $D \triangleq \frac{\sum_{z=1}^{Z} D_{z}}{Z}$.

\footnotetext{
${ }^{2}$ We do not consider a wireless network with multiple channels, therefore,
} we omit the channel assignment procedure of DMDS in this work. 


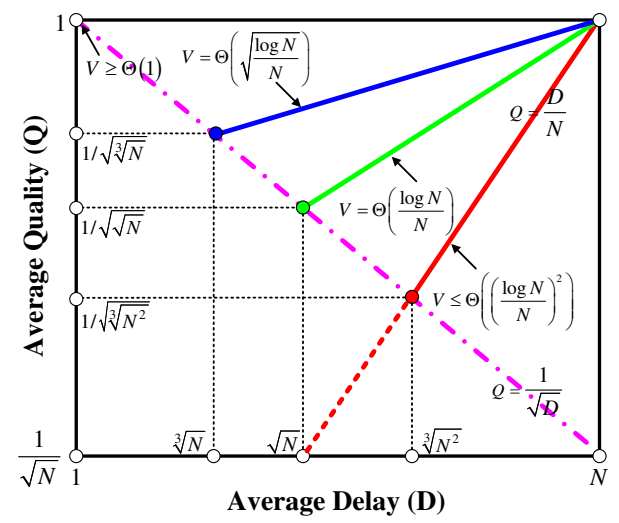

Fig. 3. Quality-Delay scaling tradeoff (in log-log scale) for different velocities.

A graphical representation of our results is presented in Fig. 3. From the displayed result, we can observe that a wide range of quality-delay tradeoff is possible for $V<\Theta(1)$ in the framework of DMDS. For a given $V$, the feasible combinations of $Q$ and $D$ situate on a line departing from the common point $D=N, Q=1$ (which represents the maximum delay and the best quality). The little circles, which situate on the line $Q=1 / \sqrt{D}$, denote the smallest achievable delay for each $V$ (corresponding to the worst quality). We can also observe that, when $0 \leq V \leq \Theta\left(\left(\frac{\log 2 N}{2 N}\right)^{2}\right)$, all points situate on the line $Q=\frac{D}{N}$. For $V \geq \Theta(1)$ no quality-delay tradeoff is achievable since the quality is maximized in conjunction with the minimization of delay.

\section{TheOretical ANALYSis FOR QuAlity AND DELAY}

Theorem 1: Let $N$ be the number of nodes in wireless ad hoc networks, and $V$ be the each node's mobility velocity. Using DMDS with the RWMM assumption, a wide range of quality-delay tradeoff is possible for any $0 \leq V<\Theta(1)$ (shown in Fig. 3), in particular, the minimal values of $Q$ and $D$ satisfy $Q=1 / \sqrt{D}$.

The proof of this theorem proceeds as follows. First we study the communication probability (Lemma 1) and hop numbers (Lemma 2) for any two nodes. Then, Lemma 3 and Lemma 4 give lower bounds on the quality and delay expectation value for each velocity, which implies (Corollary 1) that there is a relationship between each bound of quality and delay. We also show (Lemma 5) that there exists an achievable quality-delay tradeoff range for each velocity and this range is a linear function (Corollary 2).

We start by analyzing the probability of two nodes can communicate with each other. Let the probability of each node can communicate with any other nodes is $\mathbb{P}_{c}(N, V, R)$, where $R$ is the each node's transmission range, then we have the following lemma.

Lemma 1: If $\xi=\frac{\sum_{k=1}^{K} p_{l, k}}{K \sqrt{V+R} \log N}$, the value of $\mathbb{P}_{c}(N, V, R)$ satisfies:

$$
\mathbb{P}_{c}(N, V, R) \geq e^{-\xi} .
$$

Proof: Since this proof is similar to that of $[6$, Theorem 14], we omit it here.
Lemma 2: Assume that $N$ and $R$ are known, for any $V \geq 0$, let

$$
\begin{gathered}
\omega_{1}(V)=\left\lceil\frac{\omega_{2}(V)}{\mathbb{P}_{c}(N, V, R)}\right\rceil, \\
\omega_{2}(V)=\frac{K}{\log N \sqrt{V+R}} / \sum_{k=1}^{K} \frac{\lambda_{k} W_{k}}{D_{k}},
\end{gathered}
$$

then under the DMDS with RWMM, the transmission delay from source to destination is finite, and the average number of the hops is not larger than $\left|\omega_{1}(V)\right|$.

Outline of the proof: We first introduce a theorem introduced in [7]: Let $\left\{h_{m, n}\right\}$ be a set of random variables satisfying $0 \leq m<n$. Suppose that $\left\{h_{m, n}\right\}$ has the following five properties:

1) $h_{0, n} \leq h_{0, m}+h_{m, n}$;

2) for each $n, \mathbb{E}\left(\left|h_{0, n}\right|\right)<\infty$ and $\mathbb{E}\left(h_{0, n}\right) \geq c n$ for some constant $c>-\infty$;

3) the distribution of $\left\{h_{m, m+k}: k \geq 1\right\}$ does not depend on $m$;

4) for each $k \geq 1,\left\{h_{n k, n k+k}: n \geq 0\right\}$ is a stationary sequence;

5) The stationary sequence in 4) is ergodic.

We can get: i) $\rho \triangleq \lim _{n \rightarrow \infty} \frac{\mathbb{E}\left[h_{0, n}\right]}{n}=\inf _{n \geq 1} \frac{\mathbb{E}\left[h_{0, n}\right]}{n}$; ii) $H \triangleq$ $\lim _{n \rightarrow \infty} \frac{h_{0, n}}{n}$ exists; iii) $\mathbb{E}[H]=\rho$; iv) $H=\rho$.

Then, in order to apply the previous theorem to our problem, we make the following notations. Let $h_{m, n}$ and $H_{m, n}$ be the multi-hop (maybe single-hop) delay and the number of hops from node $m$ to node $n$, respectively. By the characteristics of DMDS and RWMM, it is obvious that conditions 1), 3) and 4) hold for $\left\{h_{m, n}\right\}$. We only need to show condition 2) and 5) also hold for $\left\{h_{m, n}\right\}$. The following procedure is same with in [7, Theorem 2].

Lemma 3: Assume that $N$ and $R$ are known, let $Q$ denote the video quality and $0 \leq V<\Theta(1)$. Using DMDS with the RWMM, the expectation value of $Q$ is related to $V$, and there exists a constant $B_{Q}\left(B_{Q} \in \mathbb{R}_{+}\right)$enabling

$$
\mathbb{E}[Q(V)] \geq B_{Q}, \forall V \in[0, \Theta(1)) .
$$

Similar to [6], we define a potential function $\Phi(q)=\sum_{n=1}^{N}\left(Q_{n}-Q\right)$ and a variance function $v_{n}(q)=\frac{1}{N} \sum_{n^{\prime} \neq n}^{n=1}\left(Q_{n}-Q_{n^{\prime}}\right)$. In order to prove Lemma 3, we should use the following propositions.

Proposition 1: $\forall V^{\prime}, V \in[0, \Theta(1))$, $\sum_{n=1}^{N}\left(\mathbb{E}\left[Q_{n}\left(V^{\prime}\right)\right] \mid Q(V)=q\right)^{2}=N Q^{2}+\sum_{n=1}^{N} v_{n}(q)^{2}$

$$
\begin{aligned}
& \text { Proof: } \\
& \sum_{n=1}^{N}\left(\mathbb{E}\left[Q_{n}\left(V^{\prime}\right)\right] \mid Q(V)=q\right)^{2}=\sum_{n=1}^{N}\left(Q+v_{n}(q)\right)^{2} \\
& =N Q^{2}+2 Q \sum_{n=1}^{N} v_{n}(q)+\sum_{n=1}^{N} v_{n}(q)^{2},
\end{aligned}
$$

where the second term is zero due to the definition of variance function.

Proposition 2: $\forall V^{\prime}, V \in[0, \Theta(1))$, there exists a constant $B_{Q}\left(B_{Q} \in \mathbb{R}_{+}\right)$,

$$
\mathbb{E}\left[\Phi\left(Q\left(V^{\prime}\right)\right) \mid Q(V)=q\right] \geq B_{Q} .
$$


Proof:

$$
\begin{aligned}
\text { LHS of }(8) & =\sum_{n=1}^{N} \mathbb{E}\left[Q_{n}\left(V^{\prime}\right)^{2} \mid Q(V)=q\right]-N Q^{2} \\
& =\sum_{n=1}^{N}\left(\mathbb{E}\left[Q_{n}\left(V^{\prime}\right) \mid Q(V)=q\right]\right)^{2} \\
& +\sum_{n=1}^{N} \operatorname{var}\left(Q_{n}\left(V^{\prime}\right) \mid Q(V)=q\right) .
\end{aligned}
$$

Using the Observation 3.3 of [8], we can get the result.

Proof of Lemma 3: From Propositions 1, 2, we can get the result of Lemma 3.

Lemma 4: Assume that $N$ and $R$ are known, let $D$ denote the transmission delay which is defined in Section II and $0 \leq$ $V<\Theta(1)$. Using DMDS with RWMM, the expectation value of $D$ is related to $V$, and there exists a constant $B_{D}\left(B_{D} \in\right.$ $\mathbb{R}_{+}$) enabling

$$
\mathbb{E}[D(V)] \geq B_{D}, \forall V \in[0, \Theta(1)) .
$$

Proof: Since the proof process is similar to that of Lemma 3, we do not repeat it here.

Corollary 1: Assume that $N$ and $R$ are known, for $0 \leq$ $V<\Theta(1)$, using DMDS with RWMM, all the lower bounds of $Q$ and $D$ lie on one curve.

Lemma 5: Assume that $N$ and $R$ are known, let $Q$ and $D$ denote the video quality and transmission delay. Using DMDS with RWMM, a wide range of $Q-D$ tradeoff is possible for any $0 \leq V<\Theta(1)$,

Proof: See Appendix.

Then we have the following corollary to describe the $Q-D$ tradeoff range.

Corollary 2: For a given $V \in[0, \Theta(1))$, the feasible combinations of $Q$ and $D$ lie on a line departing from the common point $D=N, Q=1$.

Now we can give the proof of Theorem 1 .

Proof of Theorem 1: For a fixed $\eta>0$, when $0 \leq V<$ $\Theta(1)$, we have:

$$
\begin{aligned}
\mathbb{P}(Q>q) & =\mathbb{P}(Q>q ; N \leq q(1-\eta)) \\
& +\mathbb{P}(Q>q ; N>q(1-\eta)) \\
& <\mathbb{P}\left(\sum_{n=1}^{\lfloor q(1-\eta)\rfloor} Q_{n}>q\right)+\mathbb{P}(N>q(1-\eta)) .
\end{aligned}
$$

Also, we can have a lower bound:

$$
\begin{aligned}
\mathbb{P}(Q>q) & =\mathbb{P}(Q>q ; N>q(1+\eta)) \\
& =\mathbb{P}(N>q(1+\eta))-\mathbb{P}(Q \leq q ; N>q(1+\eta)) \\
& \geq \mathbb{P}(N>q(1+\eta))-\mathbb{P}\left(\sum_{n=1}^{\lfloor q(1+\eta)\rfloor} Q_{n} \leq q\right) .
\end{aligned}
$$

Specifically, using the Chernoff bound, we can show that there exists constants $\kappa, \iota$ such that

$$
\mathbb{P}\left(\sum_{n=1}^{\lfloor q(1-\eta)\rfloor} Q_{n}>q\right)<\kappa e^{-q \iota} .
$$

Thus, as $q \rightarrow \infty$, it follows that

$$
\begin{aligned}
& \mathbb{P}\left(\sum_{n=1}^{\lfloor q(1-\eta)\rfloor} Q_{n}>q\right)=o(\mathbb{P}(N>q)), \\
& \mathbb{P}\left(\sum_{n=1}^{\lfloor q(1-\eta)\rfloor} Q_{n} \leq q\right)=o(\mathbb{P}(N>q)) .
\end{aligned}
$$

Go back to (10), we get

$$
\begin{aligned}
\limsup _{q \rightarrow \infty} \frac{\mathbb{P}(Q>q)}{\mathbb{P}(N>q)} & \leq \limsup _{q \rightarrow \infty} \frac{\mathbb{P}\left(\sum_{n=1}^{\lfloor q(1-\eta)\rfloor} Q_{n}>q\right)}{\mathbb{P}(N>q)} \\
& +\limsup _{q \rightarrow \infty} \frac{\mathbb{P}(N>q(1-\eta))}{\mathbb{P}(N>q)} .
\end{aligned}
$$

According to (11), the first term of (13) is zero, so that for all $\eta$, we have

$$
\limsup _{q \rightarrow \infty} \frac{\mathbb{P}(Q>q)}{\mathbb{P}(N>q)} \leq \limsup _{q \rightarrow \infty} \frac{\mathbb{P}(N>q(1-\eta))}{\mathbb{P}(N>q)} .
$$

Taking the limit $\eta \downarrow 0$, we can get

$$
\limsup _{q \rightarrow \infty} \frac{\mathbb{P}(Q>q)}{\mathbb{P}(N>q)} \leq \lim _{\eta \downarrow 0} \limsup _{q \rightarrow \infty} \frac{\mathbb{P}(N>q(1-\eta))}{\mathbb{P}(N>q)}=1 .
$$

Similarly, we can also get:

$$
\limsup _{q \rightarrow \infty} \frac{\mathbb{P}(Q>q)}{\mathbb{P}(N>q)} \geq 1 .
$$

(15) and (16) imply the results.

\section{Conclusions}

In this paper, we study the impact of mobility on video streaming over wireless ad hoc networks from the perspectives of video quality and transmission delay. In particular, our contributions are twofold. First, we have investigated the optimal node velocity for for the mobile video network under the random walk mobility model. This strategy helps to identify the contribution of mobility in the improvement the performance of video communications. Second, we present the achievable quality-delay tradeoff range for any node velocity. This point is very helpful for the network operators to design an appropriate quality or delay requirement for general wireless video transmission.

\section{ACKNOWLEDGEMENTS}

This work is supported by A Project Funded by the Priority Academic Program Development of Jiangsu Higher Education Institutions-Information and Communication Engineering. 


\section{APPENDIX: PROOF OF LEMMA 5}

We first get an upper bound for the tradeoff when $0 \leq V<$ $\Theta(1)$. For every $\eta>0$, we have

$$
\begin{aligned}
& \mathbb{P}\left(\left|\omega_{1}\right| \geq q^{B_{Q} / B_{D}} ; Q \geq q\right)= \\
& \mathbb{P}\left(\left|\omega_{1}\right| \geq q^{B_{Q} / B_{D}} ; Q \geq q ; Q_{n} \leq q(1-\eta)\right)+ \\
& \mathbb{P}\left(\left|\omega_{1}\right| \geq q^{B_{Q} / B_{D}} ; Q \geq q ; Q_{n}>q(1-\eta)\right) \stackrel{(a)}{\leq} \\
& \mathbb{P}\left(Q \geq q ; Q_{n} \leq q(1-\eta)\right)+ \\
& \mathbb{P}\left(\left|\omega_{1}\right| \geq q^{B_{Q} / B_{D}}+q(1-\eta)\right) \stackrel{(b)}{<} \\
& \mathbb{P}\left(\sum_{n=1}^{\lfloor q(1-\eta)\rfloor} Q_{n}>q\right)+ \\
& \mathbb{P}\left(\left|\omega_{1}\right| \geq q^{B_{Q} / B_{D}}+q(1-\eta)\right) .
\end{aligned}
$$

In $(a)$, we use Lemma 3, and in (b), we useLemma 4. Next, we can derive a lower bound:

$$
\begin{aligned}
& \mathbb{P}\left(\left|\omega_{1}\right| \geq q^{B_{Q} / B_{D}} ; Q \geq q\right) \geq \\
& \mathbb{P}\left(\left|\omega_{1}\right| \geq q^{B_{Q} / B_{D}} ; Q \geq q ; Q_{n}>q(1+\eta)\right)= \\
& \mathbb{P}\left(\left|\omega_{1}\right| \geq q^{B_{Q} / B_{D}} ; Q_{n}>q(1+\eta)\right)- \\
& \mathbb{P}\left(\left|\omega_{1}\right| \geq q^{B_{Q} / B_{D}} ; Q<q ; Q_{n}>q(1+\eta)\right) \geq \\
& \mathbb{P}\left(\left|\omega_{1}\right| \geq q^{B_{Q} / B_{D}}+q(1+\eta)\right)- \\
& \mathbb{P}\left(\sum_{n=1}^{\lfloor q(1+\eta)\rfloor} Q_{n} \leq q\right) .
\end{aligned}
$$

We can observe that the terms $\mathbb{P}\left(\sum_{n=1}^{\lfloor q(1-\eta)\rfloor} Q_{n}>q\right)$ in (A1) and $\mathbb{P}\left(\sum_{n=1}^{\lfloor q(1+\eta)\rfloor} Q_{n} \leq q\right)$ in (A2) decay exponentially fast as $q \rightarrow \infty$ for any $\eta>0$. This is because each link calculates the rates to achieve a balance between the rate increment and network congestion, and each source determines the optimal path distribution to achieve minimum video distortion in the framework DMDS. More precisely, a Chernoff bound can be used to get

$$
\mathbb{P}\left(\sum_{n=1}^{\lfloor q(1-\eta)\rfloor} Q_{n}>q\right)=o\left(\mathbb{P}\left(\left|\omega_{1}\right| \geq q^{B_{Q} / B_{D}}+q\right)\right),
$$

and

$$
\mathbb{P}\left(\sum_{n=1}^{\lfloor q(1-\eta)\rfloor} Q_{n} \leq q\right)=o\left(\mathbb{P}\left(\left|\omega_{1}\right| \geq q^{B_{Q} / B_{D}}+q\right)\right) .
$$

Case I: $B_{Q} / B_{D}<1$. Using (A1), we have

$$
\begin{aligned}
\limsup _{q \rightarrow \infty} \frac{\mathbb{P}\left(\left|\omega_{1}\right| \geq q^{B_{Q} / B_{D}} ; Q \geq q\right)}{\mathbb{P}\left(\left|\omega_{1}\right| \geq q\right)} \leq \\
\limsup _{q \rightarrow \infty} \frac{\mathbb{P}\left(\sum_{n=1}^{\lfloor q(1-\eta)\rfloor} Q_{n}>q\right)}{\mathbb{P}\left(\left|\omega_{1}\right| \geq q\right)}+ \\
\limsup _{q \rightarrow \infty} \frac{\mathbb{P}\left(\left|\omega_{1}\right| \geq q^{B_{Q} / B_{D}}+q(1-\eta)\right)}{\mathbb{P}\left(\left|\omega_{1}\right| \geq q\right)} .
\end{aligned}
$$

Since $B_{Q} / B_{D}<1, \forall \eta>0$ we can get $\limsup _{q \rightarrow \infty} \frac{\mathbb{P}\left(\left|\omega_{1}\right| \geq q^{B_{Q} / B_{D}} ; Q \geq q\right)}{\mathbb{P}\left(\left|\omega_{1}\right| \geq q\right)} \leq \limsup _{q \rightarrow \infty} \frac{\mathbb{P}\left(\left|\omega_{1}\right| \geq q(1-\eta)\right)^{8]}}{\mathbb{P}\left(\left|\omega_{1}\right| \geq q\right)} . \begin{aligned} & \text { P. Berenbrink, T. Friedetzky, L. A. Goldberg, P. Goldberg, Z. Hu, and } \\ & \text { R. Martin, "Distributed Selfish Load Balancing," in Proc. of SODA } \\ & \text { 2006. }\end{aligned}$
Thus,

$$
\begin{aligned}
& \limsup _{q \rightarrow \infty} \frac{\mathbb{P}\left(\left|\omega_{1}\right| \geq q^{B} Q^{/ B_{D}} ; Q \geq q\right)}{\mathbb{P}\left(\left|\omega_{1}\right| \geq q\right)} \leq \leq \\
& \lim _{\eta \downarrow 0} \limsup _{q \rightarrow \infty} \frac{\mathbb{P}\left(\left|\omega_{1}\right| \geq q(1-\eta)\right)}{\mathbb{P}\left(\left|\omega_{1}\right| \geq q\right)} \stackrel{(c)}{=} 1,
\end{aligned}
$$

where $(c)$ comes from Lemma 2. Similarly, we can use (A2) and (A4) to get

$$
\begin{aligned}
\limsup _{q \rightarrow \infty} \frac{\mathbb{P}\left(\left|\omega_{1}\right| \geq q^{B} Q^{/ B} ; Q \geq q\right)}{\mathbb{P}\left(\left|\omega_{1}\right| \geq q\right)} \geq & \\
& \lim _{\eta \downarrow 0} \limsup _{q \rightarrow \infty} \frac{\mathbb{P}\left(\left|\omega_{1}\right| \geq q(1+2 \eta)\right)}{\mathbb{P}\left(\left|\omega_{1}\right| \geq q\right)}=1 .
\end{aligned}
$$

In fact, (A5) and (A6) imply that

$$
\mathbb{P}\left(\left|\omega_{1}\right| \geq q^{B_{Q} / B_{D}} ; Q \geq q\right) \sim \mathbb{P}\left(\left|\omega_{1}\right| \geq q\right) .
$$

Case II: $B_{Q} / B_{D}=1$. We can easily get (A7).

Case III: $B_{Q} / B_{D}>1$. For the upper bound, from (A1) we can have

$$
\begin{aligned}
\limsup _{q \rightarrow \infty} \frac{\mathbb{P}\left(\left|\omega_{1}\right| \geq q^{B_{Q} / B} ; Q \geq q\right)}{\mathbb{P}\left(\left|\omega_{1}\right| \geq q^{B{ }^{/ B} D}\right)} \leq \\
\limsup _{q \rightarrow \infty} \frac{\mathbb{P}\left(\left|\omega_{1}\right| \geq q^{B_{Q} / B^{B}}+q(1-\eta)\right)}{\mathbb{P}\left(\left|\omega_{1}\right| \geq q^{B_{Q} / B_{D}}\right)} \leq 1 .
\end{aligned}
$$

Similarly, for the lower bound, from (A2), we can get

$$
\begin{aligned}
& \limsup _{q \rightarrow \infty} \frac{\mathbb{P}\left(\left|\omega_{1}\right| \geq q^{B_{Q} / B_{D}} ; Q \geq q\right)}{\mathbb{P}\left(\left|\omega_{1}\right| \geq q^{B_{Q} / B_{D}}\right)} \geq \\
& \limsup _{q \rightarrow \infty} \frac{\mathbb{P}\left(\left|\omega_{1}\right| \geq q^{B_{Q} / B_{D}}+q(1+\eta)\right)}{\mathbb{P}\left(\left|\omega_{1}\right| \geq q^{B_{Q} / B_{D}}\right)} \leq 1 .
\end{aligned}
$$

Thus, $\forall \eta>0$, we have

$$
\begin{aligned}
\limsup _{q \rightarrow \infty} \frac{\mathbb{P}\left(\left|\omega_{1}\right| \geq q^{B_{Q} / B_{D}} ; Q \geq q\right)}{\mathbb{P}\left(\left|\omega_{1}\right| \geq q^{B_{Q} / B_{D}}\right)} \geq \\
\limsup _{q \rightarrow \infty} \frac{\mathbb{P}\left(\left|\omega_{1}\right| \geq q^{B_{Q} / B_{D}}(1+\eta)\right)}{\mathbb{P}\left(\left|\omega_{1}\right| \geq q^{B_{Q} / B_{D}}\right)}=1 .
\end{aligned}
$$

So, we can get that (A7) holds for $B_{Q} / B_{D}>1$. Therefore, Lemma 5 is now proved.

\section{REFERENCES}

[1] L. Shu, M. Hauswirth, H. Chao, M. Chen, Y. Zhang, "NetTopo: A Framework of Simulation and Visualization for Wireless Sensor Networks," Ad Hoc Networks, vol 9, no. 5, pp. 799-820, 2011.

[2] L. Shu, M. Hauswirth, Y. Zhang, J. Ma, G. Min, Y.'Wang, "Cross Layer Optimization on Data Gathering in Wireless Multimedia Sensor Networks within Expected Network Lifetime," Journal of Universal Computer Science, vol. 16, no. 10, pp. 1343-1367, 2010.

[3] L. Zhou, X. Wang, W. Tu, G. Mutean, and B. Geller, "Distributed Scheduling Scheme for Video Streaming over Multi-Channel MultiRadio Multi-Hop Wireless Networks," IEEE Journal on Selected Areas in Communications, vol. 28, no. 3, pp. 409-419, 2010.

[4] H.-P. Shiang and M. van der Schaar, "Informationally Decentralized Video Streaming over Multi-hop Wireless Networkss," IEEE Transactions on Multimedia, vol. 9, no. 6, pp. 1299-1313, 2007.

[5] L. Zhou, N. Xiong, L. Shu, A. Vasilakos and S.-S. Yeo, "Context-Aware Middleware for Multimedia Service in Heterogeneous Networks," IEEE Intelligent Systems, vol. 25, no. 2, pp. 40-47, 2010.

[6] L. Ying, S. Yang and R. Srikant, "Optimal Delay-Throughput Tradeoffs in Mobile Ad Hoc Networks," IEEE Transactions on Information Theory, vol. 54, no. 9, pp. 4119-4143, 2008.

[7] W. Ren, Q. Zhao, and A. Swami "On the Connectivity and Multihop Delay of Ad Hoc Cognitive Radio Networ 